\title{
Symmetric Identities Involving q-Frobenius-Euler Polynomials under Sym (5)
}

\author{
Serkan Araci ${ }^{1, *}$, Ugur Duran², Mehmet Acikgoz ${ }^{2}$ \\ ${ }^{1}$ Department of Economics, Faculty of Economics, Administrative and Social Sciences, Hasan Kalyoncu University, Gaziantep, \\ TURKEY \\ ${ }^{2}$ University of Gaziantep, Faculty of Science and Arts, Department of Mathematics, Gaziantep, TURKEY \\ *Corresponding author: mtsrkn@hotmail.com
}

Received March 09, 2015; Revised May 23, 2015; Accepted August 28, 2015

\begin{abstract}
Following the definition of $q$-Frobenius-Euler polynomials introduced in [3], we derive some new symmetric identities under sym (5), also termed symmetric group of degree five, which are derived from the fermionic $p$-adic $q$-integral over the $p$-adic numbers field.
\end{abstract}

Keywords: Symmetric identities, q-Frobenius-Euler polynomials, Fermionic p-adic q-integral on $\mathbb{Z}_{p}$ Invariant under $S_{5}$

Cite This Article: Serkan Araci, Ugur Duran, and Mehmet Acikgoz, "Symmetric Identities Involving $q$ Frobenius-Euler Polynomials under Sym (5)." Turkish Journal of Analysis and Number Theory, vol. 3, no. 3 (2015): 90-93. doi: 10.12691/tjant-3-3-5.

\section{Introduction}

As it is known, the Frobenius-Euler polynomials $H_{n}(x)$ for $\lambda \in \mathbb{C}$ with $|\lambda|>1$ are defined by means of the power series expansion at $t=0$

$$
\sum_{n=0}^{\infty} H_{n}(x) \frac{t^{n}}{n !}=\frac{1-\lambda}{e^{t}-\lambda} e^{x t}
$$

Taking $\mathrm{x}=0$ in the Eq. (1.1), we have $H_{n}(0):=H_{n}$ that is widely known as $n$-th Frobenius-Euler number $\mathrm{cf}$. $[3,4,5,8,17,18,21]$.

Let $\mathrm{p}$ be chosen as a fixed odd prime number. Throughout this paper, we make use of the following notations: $\mathbb{Z}_{p}$ denotes topological closure of $\mathbb{Z}, \mathbb{Q}$ denotes the field of rational numbers, $\mathbb{Q}_{p}$ denotes topological closure of $\mathbb{Q}$, and $\mathbb{C}_{p}$ indicates the field of $p$-adic completion of an algebraic closure of $\mathbb{Q}_{p}$. Let $\mathbb{N}$ be the set of natural numbers and $\mathbb{N}^{*}=\mathbb{N} \cup\{0\}$.

For $d$ an odd positive number with $(p, d)=1$, let

$$
X:=X_{d}=\lim _{\bar{n}} \mathbb{Z} / d p^{N} \mathbb{Z} \text { and } X_{1}=\mathbb{Z}_{p}
$$

and

$$
a+d p^{N} \mathbb{Z}_{p}=\left\{x \in X \mid x \equiv a\left(\bmod d p^{N}\right)\right\}
$$

where $a \in \mathbb{Z}$ lies in $0 \leq a \leq d p^{N}$. See, for details, [1,2,3,4,6-17].
The normalized absolute value according to the theory of $p$-adic analysis is given by $|p|_{p}=p^{-1} \cdot q$ can be considered as an indeterminate a complex number $q \in \mathbb{C}$ with $|q|<1$, or a $p$-adic number $q \in \mathbb{C}_{p}$ with $|q-1|_{p}<p^{-\frac{1}{p-1}}$ and $q^{x}=\exp (x \log q)$ for $|x|_{p} \leq 1$. It is always clear in the content of the paper.

Throughout this paper, we use the following notation:

$$
[x]_{q}=\frac{1-q^{x}}{1-q} .
$$

which is called $q$-extension of $x$. It easily follows that $\lim _{q \rightarrow 1}[x]_{q}=x$ for any $x$.

Let $f$ be uniformly differentiable function at a point $a \in \mathbb{Z}_{p}$, which is denoted by $f \in U D\left(\mathbb{Z}_{p}\right)$. Then the $p$-adic $q$-integral on $\mathbb{Z}_{p}$ (or sometimes called $q$ Volkenborn integral) of a function $f$ is defined by Kim [10] $I_{q}(f)=\int_{\mathbb{Z}_{p}} f(x) d \mu_{q}(x)=\lim _{N \rightarrow \infty} \frac{1}{\left[p^{N}\right]_{q}} \sum_{x=0}^{p^{N}-1} f(x) q^{x}$.

It follows from the Eq. (1.3) that

$$
\begin{aligned}
& \lim _{q \rightarrow-1} I_{q}(f)=I_{-1}(f)=\int_{\mathbb{Z}_{p}} f(x) d \mu_{-1}(x) \\
& =\lim _{N \rightarrow \infty} \sum_{x=0}^{p^{N}-1} f(x)(-1)^{x} .
\end{aligned}
$$

Thus, by the Eq. (1.4), we have 


$$
I_{-1}\left(f_{n}\right)+(-1)^{n-1} I_{-1}(f)=2 \sum_{s=0}^{n-1}(-1)^{n-s-1} f(s)
$$

where $f_{n}(x)=f(x+n),(n \in \mathbb{N}$. $)$. For the applications of fermionic $p$-adic integral over the $p$-adic numbers field, see the references, e. g., $[1,2,3,4,6,7,9,11,12,16]$.

In [3], the $q$-Frobenius-Euler polynomials are defined by the following $p$-adic fermionic $q$-integral on $\mathbb{Z}_{p}$, with respect to $\mu_{-1}$ :

$$
\begin{aligned}
& H_{n, q}\left(x \mid-\lambda^{-1}\right) \\
& =\frac{\lambda+1}{2} \int_{\mathbb{Z}_{p}} \lambda^{y}[x+y]_{q}^{n} d \mu_{-1}(y) .
\end{aligned}
$$

Upon setting $\mathrm{x}=0$ into the Eq. (1.5) gives $H_{n, q}(0):=H_{n, q}$ which are called $n$-th $q$-Frobenius-Euler number.

By letting $q \rightarrow 1^{-}$in the Eq. (1.5), it yields to

$$
\begin{aligned}
& \lim _{q \rightarrow 1^{-}} H_{n, q}\left(x \mid-\lambda^{-1}\right):=H_{n}\left(x \mid-\lambda^{-1}\right) \\
& =\frac{\lambda+1}{2} \int_{\mathbb{Z}_{p}} \lambda^{y}(x+y)^{n} d \mu_{-1}(y) .
\end{aligned}
$$

Recently, many mathematicians have studied the symmetric identities on some special polynomials, see, for details, [1,6,7,9,12]. Some of mathematicians also investigated some applications of Frobenius-Euler numbers and polynomials (or its $q$-analog) cf. [3,4,5,13,14,15,16]. Moreover, Frobenius-Euler numbers at the value $\lambda=-1$ in (1.1) are Euler numbers that is closely related to Bernoulli numbers, Genocchi numbers, etc. For more information about these polynomials, look at [1-21] and the references cited therein.

In the present paper, we obtain not only new but also some interesting identities which are derived from the fermionic $p$-adic $q$-integral over the $p$-adic numbers field. The results derived here is written under Sym (5).

\section{Symmetric Identities Involving q-Frobenius-Euler Polynomials}

For $w_{i} \in \mathbb{N}$ with $w_{i}=1(\bmod 2)$ with $i \in\{1,2,3,4,5\}$, by the Eqs. (1.3) and (1.5), we obtain

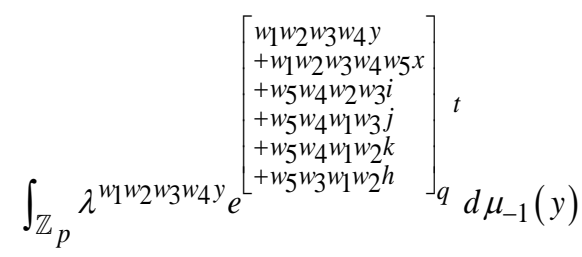

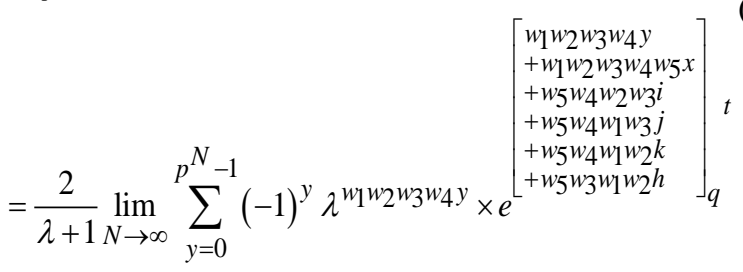

$$
\begin{gathered}
=\frac{2}{\lambda+1} \lim _{N \rightarrow \infty} \sum_{l=0}^{w_{5}-1} \sum_{y=0}^{N}(-1)^{l+y} \lambda^{w_{1} w_{2} w_{3} w_{4}\left(l+w_{5} y\right)} \\
\times e^{\left[\begin{array}{l}
w_{1} w_{2} w_{3} w_{4}\left(l+w_{5} y\right)+w_{1} w_{2} w_{3} w_{4} w_{5} x \\
+w_{5} w_{4} w_{2} w_{3} i+w_{5} w_{4} w_{1} w_{3} j \\
+w_{5} w_{4} w_{1} w_{2} k+w_{5} w_{3} w_{1} w_{2} h
\end{array}\right]_{q} .} .
\end{gathered}
$$

Taking

$$
\frac{\lambda+1}{2} \sum_{i=0}^{w_{1}-1} \sum_{j=0}^{w_{2}} \sum_{k=0}^{-1} \sum_{h=0}^{w_{3}-1}\left[\begin{array}{l}
w_{4}-1 \\
(-1)^{i+j+k+h} \\
\left.\times \lambda \begin{array}{l}
w_{5} w_{4} w_{2} w_{3} i+w_{5} w_{4} w_{1} w_{3} j \\
+w_{5} w_{4} w_{1} w_{2} k+w_{5} w_{3} w_{1} w_{2} h
\end{array}\right)
\end{array}\right]
$$

on the both sides of Eq. (2.1) gives

$\frac{\lambda+1}{2} \sum_{i=0}^{w_{1}-1} \sum_{j=0}^{w_{2}} \sum_{k=0}^{-1} \sum_{h=0}^{w_{3}-1}\left[\begin{array}{l}w_{4}-1 \\ (-1)^{i+j+k+h} \\ \left(\begin{array}{l}w_{5} w_{4} w_{2} w_{3} i+w_{5} w_{4} w_{1} w_{3} j \\ +w_{5} w_{4} w_{1} w_{2} k+w_{5} w_{3} w_{1} w_{2} h\end{array}\right)\end{array}\right]$

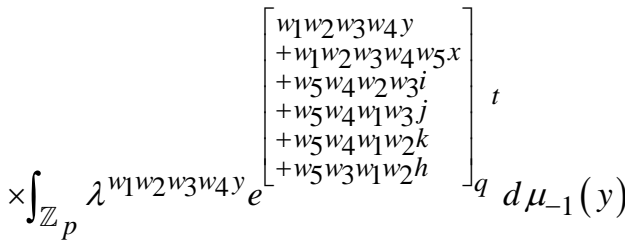

$=\lim _{N \rightarrow \infty} \sum_{i=0}^{w_{1}-1} \sum_{j=0}^{w_{2}-1} \sum_{k=0}^{w_{3}-1} \sum_{h=0}^{w_{4}-1} \sum_{l=0}^{w_{5}-1} \sum_{y=0}^{p^{N}-1}(-1)^{i+j+k+h+y+l}$

$\times \lambda^{\left[\begin{array}{l}w_{1} w_{2} w_{3} w_{4}\left(l+w_{5} y\right)+w_{5} w_{4} w_{2} w_{3} i \\ +w_{5} w_{4} w_{1} w_{3} j+w_{5} w_{4} w_{1} w_{2} k+w_{5} w_{3} w_{1} w_{2} h\end{array}\right]}$

$$
\left.\times e^{\left[\begin{array}{l}
w_{1} w_{2} w_{3} w_{4}\left(l+w_{5} y\right)+w_{1} w_{2} w_{3} w_{4} w_{5} x \\
+w_{5} w_{4} w_{2} w_{3} i+w_{5} w_{4} w_{1} w_{3} j \\
+w_{5} w_{4} w_{1} w_{2} k+w_{5} w_{3} w_{1} w_{2} h
\end{array}\right.}\right]_{q} t
$$

Note that the equation (2.2) is invariant for any permutation $\sigma \in S_{5}$. Hence, we have the following theorem.

Theorem 1. Let $w_{i} \in \mathbb{N}$ with $w_{i}=1(\bmod 2)$ with $i \in\{1,2,3,4,5\}$. Then the following

$$
\begin{aligned}
& \frac{\lambda+1}{2} \sum_{i=0}^{w_{\sigma(1)}} \sum_{j=0}^{-1 w_{\sigma(2)^{-1}}} \sum_{k=0}^{\sigma(3)^{-1}} \sum_{h=0}^{w_{\sigma}(4)^{-1}}(-1)^{i+j+k+h} \\
& \times \lambda\left(\begin{array}{c}
w_{\sigma(5)}{ }^{w} \sigma(4)^{w} \sigma(2)^{w} \sigma(3)^{i} \\
\left.+w_{\sigma(5)}\right)^{w} \sigma(4)^{w} \sigma(1)^{w} \sigma(3)^{j} \\
+w_{\sigma(5)}{ }^{w} \sigma(4)^{w} \sigma(1)^{w} \sigma(2)^{k} \\
+{ }^{k} \sigma(5)^{w} \sigma(3)^{w} \sigma(1)^{w} \sigma(2)^{h}
\end{array}\right) \\
& \times \int_{\mathbb{Z}_{p}} \lambda^{w^{w} \sigma(1)^{w_{\sigma}} \sigma(2)^{w_{\sigma}} \sigma(3)^{w} \sigma(4)}\left(l+w_{\sigma(5)} y\right) \\
& \exp \left(\left[w_{\sigma(1)} w_{\sigma(2)} w_{\sigma(3)} w_{\sigma(4)} y\right.\right. \\
& +w_{\sigma(1)} w_{\sigma(2)} w_{\sigma(3)}{ }^{w_{\sigma(4)}}{ }^{w_{\sigma(5)}}{ }^{X} \\
& w_{\sigma(5)} w_{\sigma(4)} w_{\sigma(2)} w_{\sigma(3)} i \\
& +w_{\sigma(5)} w_{\sigma(4)} w_{\sigma(1)} w_{\sigma(3)} j \\
& +w_{\sigma(5)} w_{\sigma(4)} w_{\sigma(1)} w_{\sigma(2)} k \\
& \left.\left.+w_{\sigma(5)} w_{\sigma(3)} w_{\sigma(1)} w_{\sigma(2)} h\right]_{q} t\right) d \mu_{-1}(y)
\end{aligned}
$$


holds true for any $\sigma \in S_{5}$.

By Eq. (1.2), we easily derive that

$$
\begin{gathered}
{\left[\begin{array}{l}
w_{1} w_{2} w_{3} w_{4} y+w_{1} w_{2} w_{3} w_{4} w_{5} x+w_{5} w_{4} w_{2} w_{3} i \\
+w_{5} w_{4} w_{1} w_{3} j+w_{5} w_{4} w_{1} w_{2} k+w_{5} w_{3} w_{1} w_{2} h
\end{array}\right]_{q}} \\
=\left[\begin{array}{l}
y+w_{5} x+\frac{w_{5}}{w_{1}} i \\
y
\end{array} w_{3} w_{4}\right]_{q}\left[\begin{array}{l}
w_{5} \\
+\frac{w_{2}}{w_{2}} j+\frac{w_{5}}{w_{3}} k+\frac{w_{5}}{w_{4}} h
\end{array}\right]_{q} w_{1} w_{2} w_{3} w_{4}
\end{gathered} .
$$

From Eq. (2.1) and (2.3), we obtain

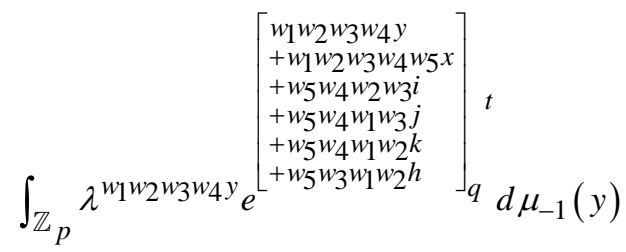

$=\sum_{n=0}^{\infty}\left[w_{1} w_{2} w_{3} w_{4}\right]_{p}^{n}$

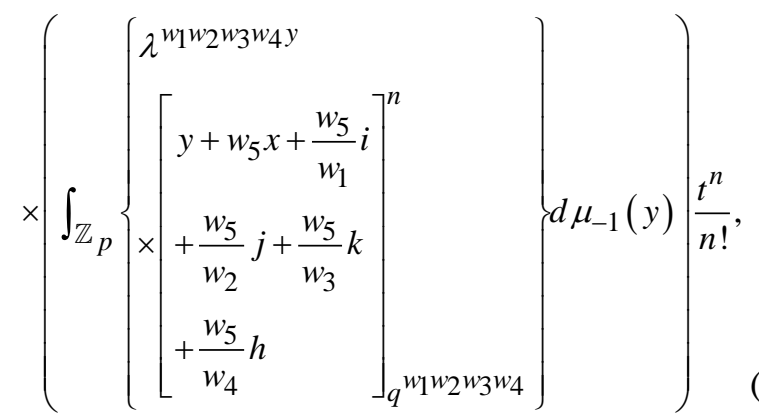

from which, we have

$$
\int_{\mathbb{Z}_{p}} \lambda^{w_{1} w_{2} w_{3} w_{4} y}\left[\begin{array}{l}
w_{1} w_{2} w_{3} w_{4} y \\
+w_{1} w_{2} w_{3} w_{4} w_{5} x \\
+w_{5} w_{4} w_{2} w_{3} i \\
+w_{5} w_{4} w_{1} w_{3} j \\
+w_{5} w_{4} w_{1} w_{2} k \\
+w_{5} w_{3} w_{1} w_{2} h
\end{array}\right]_{q}^{n} d \mu_{-1}(y)
$$

$=\frac{2}{\lambda+1}\left[w_{1} w_{2} w_{3} w_{4}\right]_{q}^{n} H_{n, q} w_{1} w_{2} w_{3} w_{4}$

$\left(w_{5} x+\frac{w_{5}}{w_{1}} i+\frac{w_{5}}{w_{2}} j+\frac{w_{5}}{w_{3}} k+\frac{w_{5}}{w_{4}} h \mid-\lambda^{-w_{1} w_{2} w_{3} w_{4}}\right)$.

$(n \geq 0)$.

Thus, by Theorem 1 and (2.5), we procure the following theorem.

Theorem 2. For $w_{i} \in \mathbb{N}$ with $w_{i}=1(\bmod 2)$ with $i \in\{1,2,3,4,5\}$, the following

$$
\begin{aligned}
& {\left[w_{\sigma(1)} w_{\sigma(2)} w_{\sigma(3)} w_{\sigma(4)}\right]_{q}^{n}} \\
& \times \sum_{i=0}^{w_{\sigma}(1)^{-1}} \sum_{j=0}^{\sigma(2)^{-1}} \sum_{k=0}^{w_{\sigma(3)^{-1}}} \sum_{h=0}^{\sigma(4)^{-1}}(-1)^{i+j+k+h} \\
& \times \lambda\left(\begin{array}{l}
{ }^{w} \sigma(5)^{w} \sigma(4)^{w} \sigma(2)^{w} \sigma(3)^{i+w_{w}} \sigma(5)^{w} \sigma(4)^{w} \sigma(1)^{w} \sigma(3)^{j} \\
+{ }^{w} \sigma(5)^{w} \sigma(4)^{w} \sigma(1)^{w} \sigma(2)^{k+w^{w}} \sigma(5)^{w} \sigma(3)^{w} \sigma(1)^{w} \sigma(2)^{h}
\end{array}\right)
\end{aligned}
$$

$$
\begin{aligned}
& \times{ }_{n, q}{ }^{w_{\sigma}}(1)^{w_{\sigma}}(2)^{w_{\sigma}}(3)^{w_{\sigma(4)}} \\
& \left(\begin{array}{c}
w_{\sigma(5)} X+\frac{w_{\sigma(5)}}{w_{\sigma(1)}} i+\frac{w_{\sigma(5)}}{w_{\sigma(2)}} j+\frac{w_{\sigma(5)}}{w_{\sigma(3)}} k \\
+\frac{w_{\sigma(5)}}{w_{\sigma(4)}} h \mid-\lambda^{-w_{\sigma(1)} w_{\sigma(2)}{ }^{w} \sigma(3)^{w}{ }^{w}(4)}
\end{array}\right)
\end{aligned}
$$

holds true for any $\sigma \in S_{5}$.

It is shown by using the definition of $[x]_{q}$ that

$$
\begin{aligned}
& {\left[y+w_{5} x+\frac{w_{5}}{w_{1}} i+\frac{w_{5}}{w_{2}} j+\frac{w_{5}}{w_{3}} k+\frac{w_{5}}{w_{4}} h\right]_{q}^{n} w_{1} w_{2} w_{3} w_{4}} \\
& \sum_{m=0}^{n}\left(\begin{array}{l}
n \\
m
\end{array}\right)\left(\frac{\left[w_{5}\right]_{q}}{\left[w_{1} w_{2} w_{3} w_{4}\right]_{q}}\right)^{n-m} \\
& \times\left[\begin{array}{l}
w_{4} w_{2} w_{3} i+w_{4} w_{1} w_{3} j \\
+w_{4} w_{1} w_{2} k+w_{3} w_{1} w_{2} h
\end{array}\right]_{q}^{n-m} \\
& \quad m\left(\begin{array}{l}
w_{5} w_{4} w_{2} w_{3} i+w_{5} w_{4} w_{1} w_{3} j \\
+w_{5} w_{4} w_{1} w_{2} k+w_{5} w_{3} w_{1} w_{2} h
\end{array}\right)\left[y+w_{5} x\right]_{q}^{m} w_{1} w_{2} w_{3} w_{4} .
\end{aligned}
$$

Taking $\int_{\mathbb{Z}_{p}} \lambda^{w_{1} w_{2} w_{3} w_{4} y} d \mu_{-1}(y)$ on the both sides of Eq.(2.6) gives

$$
\begin{gathered}
\int_{\mathbb{Z}_{p}} \lambda^{w_{1} w_{2} w_{3} w_{4} y}\left[\begin{array}{l}
y+w_{5} x+\frac{w_{5}}{w_{1}} i \\
+\frac{w_{5}}{w_{2}} j \\
\left.+\frac{w_{5}}{w_{3}} k+\frac{w_{5}}{w_{4}} h\right]_{q}^{n} d \mu_{-1}(y)
\end{array}\right. \\
=\sum_{m=0}^{n}\left(\begin{array}{l}
n \\
m
\end{array}\right)\left(\frac{\left[w_{5}\right]_{q}}{\left[w_{1} w_{2} w_{3} w_{4}\right]_{q} w_{4}}\right)^{n-m}
\end{gathered}
$$

$\times\left[w_{4} w_{2} w_{3} i+w_{4} w_{1} w_{3} j+w_{4} w_{1} w_{2} k+w_{3} w_{1} w_{2} h\right]_{q}^{n-m}$

$\times q^{m\left(w_{5} w_{4} w_{2} w_{3} i+w_{5} w_{4} w_{1} w_{3} j+w_{5} w_{4} w_{1} w_{2} k+w_{5} w_{3} w_{1} w_{2} h\right)}$

$\times \int_{\mathbb{Z}_{p}} \lambda^{w_{1} w_{2} w_{3} w_{4} y}\left[y+w_{5} x\right]_{q}^{m}{ }_{w_{1} w_{2} w_{3} w_{4}} d \mu_{-1}(y)$

$=\frac{2}{1+\lambda} \sum_{m=0}^{n}\left(\begin{array}{l}n \\ m\end{array}\right)\left(\frac{\left[w_{5}\right]_{q}}{\left[w_{1} w_{2} w_{3} w_{4}\right]_{q}}\right)^{n-m}$

$\times\left[w_{4} w_{2} w_{3} i+w_{4} w_{1} w_{3} j+w_{4} w_{1} w_{2} k+w_{3} w_{1} w_{2} h\right]_{q}^{n-m} w_{5}$

$\times q^{m\left(w_{5} w_{4} w_{2} w_{3} i+w_{5} w_{4} w_{1} w_{3} j+w_{5} w_{4} w_{1} w_{2} k+w_{5} w_{3} w_{1} w_{2} h\right)}$

$\times H_{n, q^{w_{1}} w_{2} w_{3} w_{4}}\left(w_{5} X \mid-\lambda^{-w_{1} w_{2} w_{3} w_{4}}\right)$.

By the Eq. (2.7), we have

$$
\left[w_{1} w_{2} w_{3} w_{4}\right]_{q}^{n} \frac{\lambda+1}{2} \sum_{i=0}^{w_{1}-1} \sum_{j=0}^{w_{2}-1} \sum_{k=0}^{w_{3}-1} \sum_{h=0}^{w_{4}-1}(-1)^{i+j+k+h}
$$


$\times \lambda^{\left(w_{5} w_{4} w_{2} w_{3} i+w_{5} w_{4} w_{1} w_{3} j+w_{5} w_{4} w_{1} w_{2} k+w_{5} w_{3} w_{1} w_{2} h\right)}$

$\times \int_{\mathbb{Z}_{p}} \lambda^{w_{1} w_{2} w_{3} w_{4} y}\left[\begin{array}{l}y+w_{5} x+\frac{w_{5}}{w_{1}} i \\ +\frac{w_{5}}{w_{2}} j \\ +\frac{w_{5}}{w_{3}} k+\frac{w_{5}}{w_{4}} h\end{array}\right]_{q}^{n} d \mu_{-1}(y)$

$=\sum_{m=0}^{n}\left(\begin{array}{l}n \\ m\end{array}\right)\left[w_{1} w_{2} w_{3} w_{4}\right]_{q}^{m}\left[w_{5}\right]_{q}^{n-m}$

$\times H_{n, q} w_{1} w_{2} w_{3} w_{4}\left(w_{5} x \mid-\lambda^{-w_{1} w_{2} w_{3} w_{4}}\right)$

$\times \sum_{i=0}^{w_{1}-1} \sum_{j=0}^{w_{2}} \sum_{k=0}^{-1} \sum_{h=0}^{w_{3}-1}(-1)^{i+j+k+h}$

$\times \lambda^{\left(w_{5} w_{4} w_{2} w_{3} i+w_{5} w_{4} w_{1} w_{3} j+w_{5} w_{4} w_{1} w_{2} k+w_{5} w_{3} w_{1} w_{2} h\right)}$

$\times q^{m\left(w_{4} w_{2} w_{3} i+w_{4} w_{1} w_{3} j+w_{4} w_{1} w_{2} k+w_{3} w_{1} w_{2} h\right)}$

$\times\left[w_{2} w_{4} w_{3} i+w_{1} w_{3} w_{4} j+w_{1} w_{2} w_{4} k+w_{1} w_{2} w_{3} h\right]_{q}^{n-m}$

$=\sum_{m=0}^{n}\left(\begin{array}{l}n \\ m\end{array}\right)\left[w_{1} w_{2} w_{3} w_{4}\right]_{q}^{m}\left[w_{5}\right]_{q}^{n-m}$

$\times H_{n, q} w_{1} w_{2} w_{3} w_{4}\left(w_{5} x \mid-\lambda^{-w_{1} w_{2} w_{3} w_{4}}\right)$

$\times C_{n, q} w_{5}\left(w_{1}, w_{2}, w_{3}, w_{4} \mid m\right)$,

where

$$
\begin{aligned}
& C_{n, q} w_{5}\left(w_{1}, w_{2}, w_{3}, w_{4} \mid m\right) \\
& =\sum_{i=0}^{w_{1}-1} \sum_{j=0}^{w_{2}-1} \sum_{k=0}^{w_{3}-1} \sum_{h=0}^{w_{4}-1}(-1)^{i+j+k+h} \\
& \times \lambda^{\left(w_{5} w_{4} w_{2} w_{3} i+w_{5} w_{4} w_{1} w_{3} j+w_{5} w_{4} w_{1} w_{2} k+w_{5} w_{3} w_{1} w_{2} h\right)} \\
& \times q^{m\left(w_{4} w_{2} w_{3} i+w_{4} w_{1} w_{3} j+w_{4} w_{1} w_{2} k+w_{3} w_{1} w_{2} h\right)} \\
& \times\left[w_{2} w_{4} w_{3} i+w_{1} w_{3} w_{4} j+w_{1} w_{2} w_{4} k+w_{1} w_{2} w_{3} h\right]_{q_{5} w_{5}}^{n-m} .
\end{aligned}
$$

Consequently, by (2.9), we get the following theorem.

Theorem 3. Let $w_{i} \in \mathbb{N}$ with $w_{i}=1(\bmod 2)$ with $i \in\{1,2,3,4,5\}$. Then the following expression

$$
\begin{aligned}
& \sum_{m=0}^{n}\left(\begin{array}{l}
n \\
m
\end{array}\right)\left[w_{\sigma(1)} w_{\sigma(2)} w_{\sigma(3)} w_{\sigma(4)}\right]_{q}^{m}\left[w_{5}\right]_{q}^{n-m} \\
& \times H_{n, q}^{w_{\sigma(1)} w_{\sigma(2)} w_{\sigma(3)} w_{\sigma(4)}}\left(w_{\left.\sigma(5)^{X \mid-\lambda}\left[\begin{array}{l}
-w_{\sigma(1)} w^{w} \sigma(2) \\
\times w_{\sigma(3)} w^{w}(4)
\end{array}\right]\right)}\right) \\
& \times C_{n, q}{ }^{w_{\sigma(5)}}\left(w_{\sigma(1)}, w_{\sigma(2)}, w_{\sigma(3)}, w_{\sigma(4)} \mid m\right)
\end{aligned}
$$

holds true for some $\sigma \in S_{5}$.

\section{Conclusion}

We have derived some new interesting identities of $q$-Frobenius-Euler polynomials. We also showed that these symmetric identities are written by symmetric group of degree five.

\section{References}

[1] E. Ağyüz, M. Acikgoz and S. Araci, A symmetric identity on the $q$-Genocchi polynomials of higher order under third Dihedral group $\mathrm{D}_{3}$, Proc. Jangjeon Math. Soc. 18 (2015), No. 2, pp. 177187.

[2] S. Araci, M. Acikgoz, E. ,Sen, On the extended Kim.s p-adic $q$ deformed fermionic integrals in the $p$-adic integer ring, J. Number Theory 133 (2013) 3348-3361.

[3] S. Araci, M. Acikgoz, On the von Staudt-Clausen.s theorem related to q-Frobenius-Euler number, J. Number Theory (2016).

[4] S. Araci and M. Acikgoz, A note on the Frobenius-Euler numbers and polynomials associated with Bernstein polynomials, Adv. Stud. Contemp. Math. 22 (2012), No. 3, pp. 399-406.

[5] S. Araci, E. Sen, M. Acikgoz, Theorems on Genocchi polynomials of higher order arising from Genocchi basis, Taiwanese J. Math.(2014) Vol. 18, No. 2, pp. 473-482.

[6] D. V. Dolgy, Y. S. Jang, T. Kim, H. I. Kwon, J.-J. Seo, Identities of symmetry for $q$-Euler polynomials derived from fermionic integral on $\mathbb{Z}$ p under symmetry group $\mathrm{S}_{3}$, Applied Mathematical Sciences, Vol. 8 (2014), no. 113, 5599-5607.

[7] D. V. Dolgy, T. Kim, S.-H. Rim, S.-H. Lee, Some symmetric identities for h-extension of $q$-Euler polynomials under third dihedral group $\mathrm{D}_{3}$, International Journal of Mathematical Analysis Vol. 8 (2014), no. 48, 2369-2374.

[8] Y. He and S. J. Wang, New formulae of products of the Frobenius-Euler polynomials, J. Ineq. Appl. (2014), 2014:261.

[9] Y. S. Jang, T. Kim, S.-H. Rim, J.-J. Seo, Symmetry Identities for the Generalized Higher-Order $q$-Bernoulli Polynomials under $\mathrm{S}_{3}$, International J. Math. Anal., Vol. 8 (2014), no. 38, 1873-1879.

[10] T. Kim, $q$-Volkenborn integration, Russ. J. Math. Phys., 9 (2002), no. 3, 288-299.

[11] T. Kim, Some identities on the $q$-Euler polynomials of higher order and $q$-Stirling numbers by the fermionic $p$-adic integral on $\mathbb{Z}$ p, Russian J. Math. Phys., 16, 484-491 (2009).

[12] T. Kim, $q$-Euler numbers and polynomials associated with $p$-adic $q$-integrals, Journal of Nonlinear Mathematical Physics 14 (1) 1527, (2007).

[13] T. Kim and J. J. Seo, New identities of symmetry for Carlitz.stype $q$-Bernoulli polynomials under symmetric group of degree five, International Journal of Mathematical Analysis Vol. 9, 2015, no. 35, 1707-1713.

[14] T. Kim and J. J. Seo, Some identities of symmetry for Carlitz-type $q$-Euler polynomials invariant under symmetric group of degree five, International Journal of Mathematical Analysis Vol. 9, 2015, no. 37, 1815-1822.

[15] T. Kim, Some New identities of symmetry for higher-order Carlitz $q$-Bernoulli polynomials arising from $p$-adic $q$-integral on $\mathbb{Z} p$ under the symmetric group of degree five, Applied Mathematical Sciences, Vol. 9, 2015, no. 93, 4627-4634.

[16] D. S. Kim and T. Kim, Some identities of symmetry for Carlitz qBernoulli polynomials invariant under $\mathrm{S}_{4}$, Ars Combinatoria, Vol. CXXIII, pp. 283-289, 2015.

[17] D. S. Kim and T. Kim, Some new identities of Frobenius-Euler numbers and polynomials, J. Ineq. Appl. (2012), 2012:307.

[18] Y. Simsek, Generating functions for $q$-Apostol type FrobeniusEuler numbers and polynomials, Axioms (2012), 1, 395-403.

[19] H. M. Srivastava, Some formulas for the Bernoulli and Euler polynomials at rational arguments, Math. Proc. Camb. Philos. Soc. 129, 77-84 (2000)

[20] H. M. Srivastava, Some generalizations and basic (or $q$-) extensions of the Bernoulli, Euler and Genocchi polynomials, Appl. Math. Inform. Sci. 5, 390-444 (2011).

[21] B. Y. Yasar and M. A. Özarslan, Frobenius-Euler and FrobeniusGenocchi polynomials and their dia erential equations, NTMSCI 3 (2015), No. 2, 172-180. 